\title{
COLISTIN: adult and paediatric guideline for South Africa, 2016
}

\author{
Quinten Labuschagne ${ }^{\mathrm{a}}$, Natalie Schellack ${ }^{\mathrm{a} *}$, Andries Gous ${ }^{\mathrm{a}}$, Elmien Bronkhorst ${ }^{\mathrm{a}}$, Gustav Schellack ${ }^{\mathrm{a}}$, Lindie van Tonder ${ }^{\mathrm{a}}$, Archele \\ Truter ${ }^{a}$, Caroline Smitha, Ruth Lancaster ${ }^{a}$ and Sonya Kolman ${ }^{a}$
}

\author{
aSouth African Society of Clinical Pharmacy (SASOCP), Pretoria, South Africa \\ *Corresponding author, e-mail: natalie.schellack@smu.ac.za
}

\begin{abstract}
This document was created to serve as a guideline for colistin prescribing in South Africa. Antimicrobial resistance is threatening the effective prevention and treatment of a wide variety of infections, especially those that are caused by multi-drug-resistant (MDR) gram-negative bacteria. Colistin is typically used as salvage therapy, or last-line treatment, for MDR gram-negative infections. The dosing regimen should be optimised to be therapeutic and halt the emergence of resistance, whilst minimizing toxicity. This document provides evidence-based recommendations for adult and paediatric dosing, dosing in renal impairment, and the use of combination therapy to increase synergistic effects, as well as guidelines on the reconstitution and administration of colistin. This guideline is specifically aimed at healthcare workers who routinely treat patients with MDR gram-negative infections, especially in the intensive care setting.
\end{abstract}

Keywords: Colistimethate sodium, CMS, polymyxin E, colistin guideline, multi-drug-resistant gram-negative bacteria

\begin{abstract}
Introduction
According to the World Health Organisation (WHO), antimicrobial resistance is threatening the effective prevention and treatment of a variety of infections that are on the increase. ${ }^{1}$ Antimicrobial resistance is a global threat with new mechanisms of resistance that are emerging rapidly. In addition, nosocomial infections are typically caused by bacteria that are either highly-resistant (such as methicillin-resistant Staphylococcus aureus; MRSA), or by multi-drug-resistant gram-negative bacteria (such as Acinetobacter baumannii). Carbapenem-resistance (in organisms such as Klebsiella pneumoniae and Enterobacter spp.) is emerging in South Africa and high resistance levels are currently seen in Pseudomonas aeruginosa and A. baumannii. From 2007 to 2011 there has been a decline in susceptibility to the carbapenems from $35 \%$ to $17 \%$ for $A$. baumannii. ${ }^{2}$ The inappropriate use of antibiotics in intensive care units (ICUs) is common and contributes to the poor outcomes.
\end{abstract}

Since the approval of tigecycline by the United States Food and Drug Administration (FDA), there has not been any new marketing authorisation for any novel antimicrobial agents against gram-negative infections. Many of the multi-drug resistant (MDR) gram-negative bacteria are, therefore, only sensitive to colistin.

Colistin is an old antibiotic, discovered in the late 1940s and introduced in 1959, produced by Bacillus polymyxa var. colistinus. However, after concerns were raised about reports of nephrotoxicity, the drug was withdrawn from the market in the 1970s. ${ }^{3}$ Currently there is insufficient pharmacokinetic and pharmacodynamic data on colistin, which makes it difficult to use safely and effectively in critical care patients. ${ }^{2}$ Currently, colistin often provides the only viable antimicrobial treatment option to manage these infections. There seems to be a general lack of agreement with regard to the rational use and dosing of colistin on a worldwide scale, and in South Africa there are no officially recognised or endorsed guidelines to follow.
Disagreement exists about the correct dosing and interpretation of the various dosing units since the dosing units of colistin are not standardised amongst manufacturers. Colymycine ${ }^{\oplus}$, containing colistimethate sodium (CMS), is used in South Africa, and is available in vials that are measured in international units. Colymycine ${ }^{\circledast}$ (colistin/CMS) is not registered in South Africa, but is obtainable via the Medicines and Related Substances Act, through the Section 21 application process to the Medicines Control Council (MCC). This guideline will provide South African healthcare providers with a standard approach to using colistin as colistimethate sodium (CMS), referred to as colistin (CMS), rationally and effectively, and to clear confusion and questions about this medicine (Appendix 1). Therefore, the aim of the document is to provide clear guidance and a standardised approach to the properties, dosing, reconstitution and administration of colistin (CMS), for use in both adult and paediatric patients.

\section{Overview of colistin}

\section{Properties and mechanism of action}

Colistin (CMS) is a fermentation product consisting of both polymyxin $\mathrm{E}_{1}$ and $\mathrm{E}_{2}$, and is both a cyclic polypeptide and cation at physiological $\mathrm{pH}^{3}$ CMS is the inactive prodrug which is hydrolised to colistin, which acts as a cationic detergent and damages the bacterial cytoplasmic membrane causing leaking of intracellular substances and cell death. ${ }^{4}$ Colistin displays a high binding affinity for lipopolysaccharides (LPS) molecules. The electrostatic interaction between these molecules causes a competitive displacement of the divalent cations $\left(\mathrm{Ca}^{2+}\right.$ and $\left.\mathrm{Mg}^{2+}\right)$ from phosphate groups, causing a disruption of the membrane. The end result is an increase in the permeability of the bacterial cell, leakage of the cell contents and subsequent cell death.

\section{Antibacterial activity}

Colistin (CMS) is mainly used for its activity against MDR gramnegative strains of Pseudomonas aeruginosa, Klebsiella pneumoniae, Acinetobacter baumannii and Stenotrophomonas 
maltophilia; it does not cover the Neisseria, Proteus, Serratia, Providencia, Brucella and Edwardsiella species, Pseudomonas mallei and Burkholderia cepacia. Colistin (CMS) does not cover any gram-positive bacteria or anaerobes. ${ }^{5}$

\section{Pharmacokinetics/pharmacodynamics \\ Pharmacokinetic principles}

In normal subjects, CMS has a half-life of 1-2 hours and colistin 14-19 hours. $^{6}$ Approximately $20 \%$ or less of a CMS dosage is converted in vivo to the active colistin. ${ }^{3}$ The portion of CMS that is not converted to active colistin is eliminated by tubular secretion in the kidneys. The renal clearance of CMS is related to creatinine clearance, which means excretion is decreased in renal impairment. Colistin clearance is not related to creatinine clearance, but to plasma urea and other non-renal routes that are yet to be fully elucidated. ${ }^{6}$ Neither CMS nor colistin is highly protein bound.

The CMS volume of distribution is influenced by body weight; in contrast to CMS, the volume of distribution of colistin is independent of body weight, but has been shown to decrease in febrile patients.

Because pharmacokinetic (PK) parameters of colistin cannot be calculated without estimating the fraction of CMS converted to colistin, it is difficult to assess altered PK parameters in criticallyill patients.?

In patients with decreased renal function a larger fraction of the CMS dosage is converted to colistin, which explains the decrease in clearance of formed colistin with reduced creatinine clearance and the relationship of CMS to creatinine clearance. ${ }^{8,9}$ After the first dosage, colistin levels will be both low and variable because CMS is cleared before conversion to the active drug. ${ }^{7}$ Even though colistin does not follow renal excretion itself, colistin levels are elevated in patients with renal insufficiency, possibly because of decreased elimination of CMS, leading to a higher proportion of CMS being hydrolysed to active colistin.?

In critically-ill patients, colistin reaches peak plasma concentrations seven hours after administration of CMS.7,9 A limited number of studies on CMS and colistin in patients on renal replacement therapy revealed that continuous venovenous haemodiafiltration will clear both CMS and colistin; and, in patients with intermittent haemodialysis, both CMS and colistin undergo efficient extracorporeal clearance.

Because CMS is administered as a pro-drug that is easily converted to the active compound during storage, specific analytical assays like chromatographic procedures using high performance liquid chromatography (HPLC) or liquid chromatography-tandem-mass spectrometry (LCMS) are required for accurate pharmacokinetic studies. ${ }^{9}$

\section{Pharmacodynamic principles}

Colistin exhibits concentration-dependent killing, and is rapidly bactericidal at concentrations near or above the minimum inhibitory concentration (MIC) of the causative bacteria. Frequent dosing is important as substantial regrowth has been shown to occur at 24 hours, even at concentrations up to 64 times the MIC. ${ }^{6}$ A lower likelihood of emergence of resistance was demonstrated with an 8-hourly dosing regimen. ${ }^{6,10}$ This supports the indication of combination therapy in effective treatment of nosocomial infections. The AUC:MIC ratio (i.e. the ratio of the area under the plasma-concentration-time curve to the minimum inhibitory concentration) of total and unbound colistin predicts a superior antibacterial activity to $C_{\max }: M I C\left(C_{\max }\right.$ is the maximum serum concentration) against $P$. aeruginos $a .{ }^{10}$

Colistin displays only a modest post-antibiotic effect (PAE) at very high dosages, which are usually not achieved in clinical practice.

Colistin is widely distributed to body tissues, such as kidney, liver, heart and lungs; colistin does not penetrate into cerebral spinal fluid, pleural, synovial, or pericardial fluids. The half-life of colistin in infants (including premature infants), children and adolescents is 2-3 hours. Colistin is primarily eliminated through glomerular filtration and urinary excretion by the kidneys.

\section{Adverse effects}

Drug excretion occurs primarily via the kidneys and nephrotoxicity is also the most common adverse effect. Acute tubular necrosis will manifest through increased urea and creatinine levels, pointing towards renal toxicity. Initial reports showed nephrotoxicity rates approaching 50\%. More recent reports show much lower rates in the region of $10-30 \% .^{2} \mathrm{~A}$ large retrospective cohort study from 2000 to 2007 showed incidence rates as low as $10 \% .^{11}$ The reason for the lower rates of nephrotoxicity may be due to the use of more purified preparations, better ICU monitoring and avoidance of the coadministration of other nephrotoxic drugs (e.g. the aminoglycosides and vancomycin). Risk factors for nephrotoxicity include old-age, pre-existing renal insufficiency, low albumin levels and non-steroidal anti-inflammatory drug use. Colistinassociated nephrotoxicity is both dosage-dependent and reversible. ${ }^{5}$ Measures used to prevent or limit nephrotoxicity include strict monitoring of renal function with dosage adjustments when necessary, proper hydration and limiting the co-administration of other nephrotoxic drugs. ${ }^{2}$

Neurotoxicity is less common. Symptoms include muscle weakness, vertigo, dizziness, visual disturbances, confusion, hallucinations, ataxia, seizures and neuromuscular blockade. The most common clinical effect is paraesthesia (27\%). ${ }^{5}$ Colistin interacts with neurons because of their high lipid content Triggers include hypoxia, co-administration of muscle relaxants, narcotics and/or sedatives and impaired renal function. During the 1970 s, reports of neurotoxicity was about $7 \%$, but recent retrospective studies did not find evidence of colistin-associated neurotoxic adverse events. ${ }^{12}$ Symptoms are mild, dosagedependant and resolve with the discontinuation of the drug.

\section{Dosing of colistin (CMS) \\ Parenteral dosing of colistin (CMS)}

Colistin (CMS) is currently being used as 'salvage therapy' or as a last-line treatment option for MDR gram-negative infections. Dosing regimens need to be optimised to kill the bacteria and halt the emergence of resistance and toxicity. ${ }^{3}$

An increase in the daily dosage from 2 million units (MU) to $9 \mathrm{MU}$ improved clinical cure rates from $51 \%$ to $70 \% .{ }^{13}$ By using a dosing regimen of $9 \mathrm{MU}$ as a loading dosage, followed by $4.5 \mathrm{MU}$ as a maintenance dosage, a resultant clinical cure rate of $82 \%$ was achieved.

Dosing regimens of 2-3 MU every eight hours showed maximum mean steady-state concentrations $\left(C_{\max }\right)$ of 2.21 and $2.93 \mu \mathrm{g} / \mathrm{ml}$ respectively. This may indicate that the current colistin (CMS) dosing regimen may be inadequate for infections caused by 
pathogens with a susceptibility breakpoint of $2 \mu \mathrm{g} / \mathrm{ml}$, and can result in the emergence of resistant strains. ${ }^{12}$

Synergistic effects were seen when colistin (CMS) was used in combination with the carbapenems in $77 \%$ of $A$. baumannii strains. ${ }^{14}$ An increase in the bactericidal effect was seen with colistin-carbapenem combination therapy. ${ }^{14}$ Combination antimicrobial therapy is used for maximum antimicrobial activity and to decrease the chances of resistance. ${ }^{2}$

\section{Loading dosage}

During life-threatening infections, it is important to achieve therapeutic concentrations of colistin (CMS) rapidly. Patients who are critically ill have capillary leakage, which increases their volume of distribution 4-15 fold. ${ }^{2}$ According to Landersdorfer \& Nation (2015) CMS is converted to colistin slowly. ${ }^{3}$ This fact, combined with the long half-life of formed colistin, may result in a time interval of 2-3 days to reach an adequate therapeutic plasma concentration, in the absence of a loading dosage. Therefore, it is very important to initiate colistin (CMS) therapy with a loading dosage of $12 \mathrm{MU}$ - regardless of kidney function - to reach therapeutic concentrations quicker. The high loading dosage does not affect the renal function; only the subsequent maintenance dosages would need to be adjusted.

\section{Adult dosing guideline}

The recommended dosage is based on the patient's actual body weight (ABW). However, if a patient weighs $>20 \%$ their ideal body weight (IBW), the adjusted body weight (AdjBW) should be used. If $A B W<$ IBW, use $A B W$.

Adjusted body weight, which falls between lean body weight and total body weight, is calculated as follows:

\section{Adjusted body weight $=\mathrm{LBW}+0.4(\mathrm{TBW}-\mathrm{LBW})$}

To calculate Lean Body Weight (LBW) for a MALE:

LBW $(\mathrm{kg})=50+(0.906 \times($ height in $\mathrm{cm}-152.4))$

To calculate Lean Body Weight (LBW) for a FEMALE:

LBW $(\mathrm{kg})=45+(0.906 \times($ height in $\mathrm{cm}-152.4))$
Table 1 provides the adult dosing guideline regarding treatment for MDR gram-negative infections.

Table 2 provides terminology and information regarding the vials of colistin (CMS) used in South Africa.

\section{Paediatric dosing guideline}

Table 3 illustrates the recommended dosages for paediatrics in susceptible gram-negative infections.

\section{Reconstitution and administration guideline}

Colymycine $^{\circledast}$ (Colistin/CMS) contains $1 \mathrm{MU}$ per vial. Each vial should be reconstituted with $5 \mathrm{ml}$ of $0.9 \%$ sodium chloride. Further dilute to a final volume of $50 \mathrm{ml}$ for maintenance dosages and $100 \mathrm{ml}$ for the loading dosage.

The loading dosage of $12 \mathrm{MU}$ should be given over 60 minutes.

The maintenance dosages may be given over 15-30 minutes.

Table 4 provides an outline of the reconstitution volume, diluent and infusion time to be used.

\section{Reconstitution of colistin (CMS) for use in Neonates}

For an $80 \mathrm{mg}(1 \mathrm{MU})$ vial, reconstitute with $5 \mathrm{ml} 0.9 \%$ sodium chloride (normal saline) to yield a concentration of $100000 \mathrm{lU} /$ $\mathrm{ml}$. The dosage should be infused over 30 minutes. $^{19}$

\section{Reconstitution of colistin (CMS) for use in Paediatrics}

For an $80 \mathrm{mg}(1 \mathrm{MU})$ vial, reconstitute with $2 \mathrm{ml}$ sterile water for injection to yield a concentration of $500000 \mathrm{IU} / \mathrm{ml}$. This should either be further diluted to $10 \mathrm{ml}$ with normal saline (100 $000 \mathrm{IU} /$ $\mathrm{ml}$ ) and infused over 10 minutes, or be further diluted to $50 \mathrm{ml}$ with normal saline $(20000 \mathrm{lU} / \mathrm{ml})$ and infused over 30 minutes.

\section{Renal failure and renal replacement therapy}

When the patient is in renal failure, dosage adjustments need to be made. During renal failure there is a decreased rate of CMS elimination and, in turn, more CMS is converted to colistin. This results in the impairment of colistin clearance. The CMS plasma concentration is subsequently much higher than the concentration of the formed colistin. During dialysis a large

Table 1: Recommended adult dosages of IV colistin (CMS) in critically-ill patients

\begin{tabular}{|c|c|}
\hline Normal renal function: & $\begin{array}{c}\text { Loading dose: } 12 \text { million units } \\
\text { Then: } \\
3 \text { million units every } 8 \text { hours } \\
\text { Or: } \\
4.5 \text { million units every } 12 \text { hours }\end{array}$ \\
\hline \multicolumn{2}{|l|}{ Renal impairment: } \\
\hline - $\mathrm{CrCl}^{*} 40-60 \mathrm{ml} / \mathrm{min}$ & 2 million units every 12 hours \\
\hline - $\mathrm{CrCl} * 10-40 \mathrm{ml} / \mathrm{min}$ & 2 million units every 24 hours \\
\hline - $\mathrm{CrCl}^{*}<10 \mathrm{ml} / \mathrm{min}$ & 1.5 million units every 36 hours \\
\hline \multicolumn{2}{|c|}{ Renal replacement therapy: } \\
\hline - Haemodialysis & As per $\mathrm{CrCl}^{*}$, with an additional 2 million units after dialysis \\
\hline - $C V V H D^{* *}$ & Dosing as for normal renal function \\
\hline
\end{tabular}

*Creatinine clearance (CrCL) based on Cockcroft-Gault equation;

"Continuous veno-venous hemodialysis 
Table 3: Recommended paediatric dosages for colistin (CMS)

\begin{tabular}{|c|c|}
\hline & Dosage based on Colistimethate Sodium (CMS) \\
\hline Neonates & $\begin{array}{c}50000-75000 \mathrm{IU} / \mathrm{kg} / \text { day in three divided dosages }{ }^{16,19} \\
75000-120000 \mathrm{IU} / \mathrm{kg} / \text { day in three divided dosages have been used in this } \\
\text { population }{ }^{15}\end{array}$ \\
\hline Infants and children & $75000-150000 \mathrm{IU} / \mathrm{kg} /$ day in three divided dosages. ${ }^{16,17}$ \\
\hline Inhalation CMS & $\begin{array}{l}\text { < 40kg: } 500000 \mathrm{IU} \text { every } 12 \text { hours } \\
>40 \mathrm{~kg}: 1000000 \mathrm{IU}(1 \mathrm{MU}) \text { every } 12 \text { hours } 16,20\end{array}$ \\
\hline
\end{tabular}

Table 4: Colistin (CMS) reconstitution outline information

\begin{tabular}{|l|c|c|c|}
\hline Dosage & Final volume & Diluent & Infusion time \\
\hline 12 MU loading dose & $100 \mathrm{ml}$ & $0.9 \%$ sodium chloride (normal saline) & 60 minutes \\
\hline 3 MU 8 hourly & $50-100 \mathrm{ml}$ & $0.9 \%$ sodium chloride (normal saline) & $15-30$ minutes \\
\hline $4.5 \mathrm{MU} 12$ hourly & $50-100 \mathrm{ml}$ & $0.9 \%$ sodium chloride (normal saline) & $15-30$ minutes \\
\hline
\end{tabular}

Table 5: The dosing of aerosolised colistin

\section{Body weight}

\section{$<40 \mathrm{~kg}$}

$>40 \mathrm{~kg}$

Recurrent/severe pulmonary infections
Dosing recommendation

500000 IU 12-hourly

1000000 IU 12-hourly

2000000 IU 8-hourly amount of the CMS is removed before the conversion to colistin can take place. Colistin is also subjected to extensive carriermediated tubular reabsorption in the kidneys, but dialysis does not carry this function and it passively diffuses into the dialysate. These factors imply that careful dosage selection should be done for these patients. ${ }^{3}$ Refer to Table 1 for the recommended dosages in renal failure patients.

\section{Inhaled colistin}

The administration of antibiotics via inhalation is widely used for patients with cystic fibrosis and bronchiectasis. Colistin (CMS) is the preferred drug of choice for aerosolised administration. Although there is lack of data on the efficacy and safety of inhaled colistin from randomised controlled trials, colistin (CMS) may be used as adjunctive therapy to intravenous antimicrobial treatment in critically-ill patients with ventilator-associated pneumonia (VAP) caused by MDR gram-negative bacteria, which are susceptible to colistin (CMS). ${ }^{20}$ Table 5 provides an overview of the dosing guidelines of aerolised colistin.

\section{Reconstitution and administration}

Add the required amount of colistin (CMS) to $4 \mathrm{ml}$ of $0.9 \%$ sodium chloride (normal saline) or sterile water. The solution should be nebulised at an oxygen flow rate of $8 \mathrm{l} / \mathrm{min}$ via a face mask.

\section{Side-effects of inhaled colistin}

The side-effects include bronchoconstriction (due to histamine release), coughing and a feeling of tightness in the chest and apnoea (due to neuromuscular blockade). ${ }^{20}$ To prevent bronchoconstriction and cough, treat the patient with a $\beta_{2}$ agonist before colistin inhalation. Inhaled colistin is generally well tolerated, as demonstrated by the fact that there are only minimal changes in the $\mathrm{FEV}_{1}$ before and after the inhalation. ${ }^{20}$ There is minimal renal and/or neurotoxicity with this route of administration. Good infection control measures are recommended to prevent the spread of MDR bacteria through nebulisation devices.

\section{References}

1. WHO. Antimicrobial resistance. 2015 [cited Jun 23 2015]. Available from: http://www.who.int/mediacentre/factsheets/fs194/en/.

2. Visser Kift E, Maartens G, Bamford C. Systemic review of the evidence for rational dosing of colistin. S Afr Med J. 2014;104(3):183-186.

3. Landersdorfer CB, Nation RL. Colistin: how should it be dosed for the critically ill? Critic Care Med. 2015;36:126-135.

4. Taketomo CK, Hodding JH, Kraus DM, editors. Pediatric \& neonatal dosage handbook. 21st edition. United States: Lexi-Comp. 2014; p. 541-544.

5. Yahav D, Farbman L, Leibovici L, et al. Colistin: new lessons on an old antibiotic. Clin Microbiol Inf. 2011;18:18-29.

6. Micalopoulos AS, Falagas ME. Colistin: recent data on pharmacodynamics properties and clinical efficacy in critically ill patients. Annal Inten Care. 2011;1(30):1-6

7. Ortwine JK, Kaye KS, Li J, et al. Colistin: understanding and applying recent pharmacokinetic advances. Pharmacotherapy. 2015;35(1):1116.

8. Bergen PJ, Landersdorfer $\mathrm{CB}$, Zhang J, et al. Pharmacokinetics and pharmacodynamics of "old" polymyxins: what is new? Diagn Microbiol Infect Dis. 2012;74:213-223.

9. Couet W, Gregoire N, Marchand S, et al. Colistin pharmacokinetic: the fog is lifting. Clin Microbiol Infect. 2011;2011(18):30-39.

10. Bergen PJ, Li J, Nation RL, et al. Comparison of once-twice- and thrice-daily dosing of colistin on antibacterial effect and emergence of resistance: studies with Pseudomonas aeruginosa in an in vitro pharmacodynamic model. J Antimicrob Chemother. 2008;61:636642.

11. Al Ramahi JW, Al Bashaireh A, Salman S. Colistin in critically ill patients: a critical review. Int Arab J Antimicrob Agents. 2014;4(2:2):1-15.

12. Spapen H, Jacobs R, Van Gorp V, et al. Renal and neurological side effects of colistin in critically ill patients. Annal Intens Care. 2011;1:14-20.

13. Dalfino L, Puntillo F, Mosca A, et al. High-dose, extended-Interval colistin administration in critically ill patients: is this the right dosing strategy? A preliminary study. Clin Infect Dis. 2012;54(12):1720-6.

14. Zusman O, Avni T, Leibovici L, et al. Systemic review and meta-analysis of in-vitro synergy of polymyxins and carbapenems. Antimicrob Agents Chemother. 2013;57:5104-5111.

15. Kumar P, Giri S, Shaikh F, et al. Safety and efficacy of intravenous colistin in children. Indian Pediatr. 2015;52(2):129-130. 
16. British National Formulary for children. the essential resource for clinical use of medicines in children. London: RCPCH Publication; 2014. p. P292.

17. Tamma $P$, Newland J, Pannaraj $P$, et al. The use of intravenous colistin among children in the United States. Pediatr Infect Dis J. 2013;32(1):17-22.

18. Van Saene HKF, Silvestri L, De la Cal AM. Infection control in the intensive care unit. Milan: Springer; 2005. p. 575-592.
19. Jajoo $M$, Kumar V, Jain $M$, et al. Intravenous colistin administration in neonates. Pediatr Infect Dis J. 2011;30(3):218-221.

20. Michalpulos A, Papadakis E. Inhaled anti-infective agents: emphasis on colistin. Infection. 2010;38:81-88.

Received: 18-12-2015 Accepted: 05-01-2016

\section{APPENDIX 1}

\section{COLISTIN USE/DOSAGE CHECKLIST}

\section{Approved Indications:}

Patients with a history of, suspected, or confirmed multi-drug-resistant gram-negative organism: Please check appropriate organism $\square$

\begin{tabular}{l} 
Pseudomonas aeruginosa \\
Klebsiella pneumonia \\
\hline Acinetobacter baumannii \\
\hline Carbapenem-resistant enterobacteriaceae \\
\hline
\end{tabular}

\section{Colistin dosing:}

\section{Dosing in normal patients}

- Loading dose

- Maintenance dose

$12 \mathrm{MU}$

$3 \mathrm{MU}$ tds (8-hourly)

Or 4.5 mu bd (12-hourly)

\begin{tabular}{l|c}
\hline Renal impairment: & 2 million units, 12-hourly \\
\hline - $\mathrm{CrCl}^{*} 40-60 \mathrm{ml} / \mathrm{min}$ & 2 million units, 24-hourly \\
\hline - $\mathrm{CrCl}^{*} 10-40 \mathrm{ml} / \mathrm{min}$ & 1.5 million units, 36-hourly \\
\hline - $\mathrm{CrCl}^{*}<10 \mathrm{ml} / \mathrm{min}$ & As per CrCl*, with an additional 2 million units after dialysis \\
\hline Renal replacement therapy: & Dosing as for normal renal function \\
\hline - Haemodialysis & \\
\hline - CVVHD** & \\
\hline
\end{tabular}

\section{Colistin dosing instructions:}

- Reconstitute each vial with $5 \mathrm{ml} 0.9 \%$ sodium chloride, further dilute to $100 \mathrm{ml}$ for loading dosage and $50 \mathrm{ml}$ for maintenance dosage.

- Infusion loading dose over 60 minutes.

- Infuse maintenance dose over 15-30 minutes.

- Must be given with a second agent (either rifampicin or a carbapenem) - never on its own.

\section{Additional comments:}

- Very nephrotoxic.

- Need blood results to apply for a Section 21 approval - Very important!!

- Store below 25 degrees Celsius $\left({ }^{\circ} \mathrm{C}\right)$.

- Cannot be stored once mixed - therefore discard any unused portion. 